\title{
GENERALIZATIONS OF A THEOREM OF NAMIOKA CHARLES STEGALL
}

(Communicated by J. Jerry Uhl, Jr.)

\begin{abstract}
We prove a result concerning separate and joint continuity that contains a celebrated result of Namioka.
\end{abstract}

In 1974 Namioka proved a result somewhat stronger than the following:

THEOREM I [N1]. Let $B$ be a Čech-complete topological space, $K$ a compact Hausdorff space and $f: B \times K \rightarrow M$ a separately continuous function into the metric space $M$. Then there exists a dense $G_{\delta}$ subset $G$ of $B$ such that $f$ is (jointly) continuous at each point of $G \times K$.

There is an obvious reformulation of this result. Suppose $f, B$ and $K$ are as above and $C(K)$ denotes the Banach space of continuous functions on $K$. Then the function $f$ induces a function $h: B \rightarrow C(K)$ defined by $h(b)=f(b, \cdot)$ and $h$ is continuous into $C(K)$ when $C(K)$ has the simple topology.

Our own formulation of Namioka's result, with a considerably different proof, is the following:

THEOREM II [S2]. Suppose $B$ is a Cech-complete space and $f: B \rightarrow X$ is a function into the Banach space $X$ such that for any $x^{*}$ in $X^{*}$ that is an extreme point of the unit ball of $X^{*}$ we have that $x^{*} \circ f$ is continuous. Then $f$ is normcontinuous at each point of a dense $G_{\delta}$ subset $G$ of $B$.

We postpone until the end of the paper a proof of Theorem II.

We shall say that such a function $f$ is $\sigma(X$, ext) continuous.

We shall show that a number of recent results concerning separate continuity are consequences of this theorem.

Two natural problems which have been investigated by various authors are the following:

(1) characterize those Banach spaces $X$ such that for any Baire space $B$ and any $f: B \rightarrow X$ that is weakly continuous we have that $f$ is norm-continuous at each point of a dense $G_{\delta}$ set;

(2) characterize those Baire spaces for which Namioka's theorem is always valid.

We show that in all of the known cases we need only assume in (1) that $f$ is continuous with respect to the extremal topology. We reduce a number of results concerning problems (1) and (2) to Theorem I (see Theorem 3 below).

Only Hausdorff topological spaces will be considered. For background material in topology, see [ $\mathbf{E}$ and $\mathbf{B}$ ].

Let $T$ be a topological space. We shall say that $T$ is k-analytic, written ka, if there exist a Čech-complete and Lindelöf space $C$ and an onto continuous function

Received by the editors January 5, 1986.

1980 Mathematics Subject Classification (1985 Revision). Primary 46B99; Secondary 26B05.

(C) 1988 American Mathematical Society $0002-9939 / 88 \$ 1.00+\$ .25$ per page 
$f: C \rightarrow T$. It is a special case of a theorem of Frolik that a space $C$ is Cechcomplete and Lindelöf if and only if there exist a Polish space $M$ and a function $p: C \rightarrow M$ that is perfect. A function is perfect if it is continuous, onto, closed and inverse images of compact sets are compact. A topological space $M$ is Polish if it is homeomorphic to a complete, separable metric space. We may take as the definition of a ka space the following: a topological space $T$ is ka if there exist topological spaces $C$ and $M, M$ Polish, and continuous functions $f: C \rightarrow T$ and $p: C \rightarrow M$ so that $f$ is onto and $p$ is perfect.

A perfect map $f: T \rightarrow S$ is minimal if for any proper closed subset $E$ of $T$, $f(E) \neq S$.

LEMMA 1. Suppose that $B$ is a Baire space, $X$ is a Banach space and $g: B \rightarrow X$ is $\sigma\left(X\right.$, ext) continuous. Suppose also that $E$ is Cech-complete, $G$ is a dense $G_{\delta}$ subset of $B, h_{1}: G \rightarrow E$ is continuous, $h_{2}: E \rightarrow X$ is $\sigma(X$, ext) continuous, and $h_{2} \circ h_{1}(b)=g(b)$ for $b \in G$. Then $g$ is norm-continuous at each point of a dense $G_{\delta}$ subset of $B$.

PrOOF. The hypothesis is contained in the following diagram:

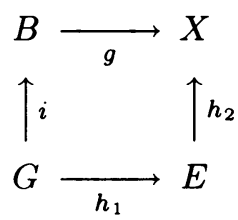

For $\varepsilon>0$, let

$$
\mathcal{U}_{\varepsilon}=\{U \mid U \text { open in } B, \operatorname{diam} g(U \cap G) \leq \varepsilon\}
$$

and

$$
U_{\varepsilon}=\bigcup\left\{U \in U_{\varepsilon}\right\}
$$

Suppose $\bar{U}_{\varepsilon} \neq B$. The closure $F$ of $h_{1}\left(\left(B \backslash \bar{U}_{\varepsilon}\right) \cap G\right)$ is also Čech-complete. Hence, by Theorem I there exists $W$ open, $W \cap F \neq \varnothing$ and $\operatorname{diam} h_{2}(W \cap F)<\varepsilon$. Since $h_{1}$ is continuous there exists $U$ open, $U \neq \varnothing, U \cap \bar{U}_{\varepsilon}=\varnothing$ and $h_{1}(U \cap G) \subseteq W$. Hence, $U$ is in $\mathcal{U}_{\varepsilon}$. Contradiction.

Let $G_{1}=G \cap \bigcap_{n=1}^{\infty} U_{1 / n}$. Then $G_{1}$ is a dense $G_{\delta}$ subset of $B$ and for any $\varepsilon>0$ and any $b \in G_{1}$ there exists $U$ open, $b \in U$ and $\operatorname{diam}(g(U \cap G))<\varepsilon$. Since balls in $X$ are $\sigma(X$, ext) closed, $\operatorname{diam} g(\overline{U \cap G}) \leq \varepsilon$. It is easy to see that $U \subseteq \overline{U \cap G}$. It follows that $g$ is norm-continuous at each point of $G_{1}$.

See [T1] for a discussion of certain compact spaces $K$ such that $C(K)$ satisfies the hypothesis of the following result.

THEOREM 2. Suppose that $X$ is a Banach space and $X$ is ka in the $\sigma(X$, ext) topology. Suppose $g: B \rightarrow X$ is a $\sigma(X$, ext) continuous function defined on the Baire space $B$. Then $g$ is norm-continuous at each point of a dense $G_{\delta}$ subset.

Proof. Let $C$ be Čech-complete and Lindelöf, let $f: C \rightarrow X$ be $\sigma(X$, ext $)$ continuous, onto and let $p: C \rightarrow M$ be perfect onto the Polish space $M$. Let

$$
D=\{(f(c), p(c)) \mid c \in C\} \subseteq X \times M .
$$

Observe that the composed mapping

$$
C \stackrel{f \times p}{\longrightarrow} D \stackrel{\text { proj }}{\longrightarrow} M
$$


is perfect, so that $f \times p: C \rightarrow D$ and proj: $D \rightarrow M$ are both perfect. Thus $D$ is also Čech-complete (see $[\mathbf{E}]$ ). Let

$$
H=\{(b, m) \mid(g(b), m) \in D\} .
$$

We have that $H$ is a closed subset of $B \times M$ and $\operatorname{proj}_{B}(H)=B$ because $D$ is closed in $X \times M$ and $f$ is onto. A consequence of the Ryll-Nardzewski, Kuratowski theorem is that there exist a dense $G_{\delta}$ subset $G$ of $B$ and a continuous function $\lambda: G \rightarrow M$ so that $H_{1}=\{(b, \lambda(b)) \mid b \in G\} \subseteq H$. Observe that $\operatorname{proj}_{B}: H_{1} \rightarrow G$ is a homeomorphism onto and we may apply Lemma 1:

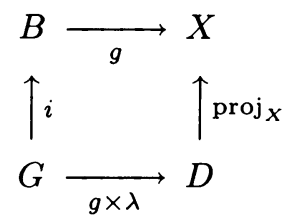

The following theorem was proved by Debs [Deb], generalizing results of St. Raymond [SR], Talagrand [T2] and Deville [Dev].

THEOREM 3. Suppose $X=C(K)$ where $K$ is a compact Hausdorff space, $g: B \rightarrow X$ is $\sigma(X$, ext) continuous and $B$ contains a dense subspace $A$ that is $k a$. Then $g$ is norm-continuous at each point of a dense $G_{\delta}$ subset of $B$.

Proof. Let $Y$ be the smallest uniformly closed subalgebra of $X$ that contains the constant functions and $g(A)$. Then $Y$ is k-analytic in the $\sigma(X, K)$ topology (see [T1]). From the Gelfand-Stone theorem, $Y$ is isometric to the $C\left(K_{1}\right)$ space where $K_{1}$ is also compact. It follows that $Y$ is closed in the $\sigma(X$, ext) topology. Thus, $g(B) \subseteq Y$ and we may apply the theorem above.

We give one multivalued version of these results.

THEOREM 4. Suppose $X$ is a Banach space that is ka in the $\sigma(X$, ext) topology and $\varphi: B \rightarrow \mathcal{P}(X)$ is a minimal upper semicontinuous compact valued (with respect to the $\sigma(X, \mathrm{ext})$ topology) multimap. Then $\{b \mid \varphi(b)$ is a point $\}$ contains a dense $G_{\delta}$ subset of $B$.

PROOF. Let

$$
G=\{(x, b) \mid x \in \varphi(b), b \in B\}
$$

be the graph of $\varphi$. Let $p: G \rightarrow B$ be projection. The following are easy: $p$ is minimal perfect, $G$ is a Baire topological space and if $H_{1}$ is a dense $G_{\delta}$ subset of $G$ then there exists a dense $G_{\delta}$ subset $H_{2} \subseteq B$ such that $p^{-1}\left(H_{2}\right) \subseteq H_{1}$. Define $f: G \rightarrow X$ by projection. There exists a dense $G_{\delta}$ subset $H$ of $G$ such that for each $(x, b) \in H, f$ is norm-continuous at $(x, b)$. If $L$ is a dense $G_{\delta}$ subset of $B$ such that $p^{-1}(L) \subseteq H$, then $\varphi(b)$ is a one-point set for $b \in L$.

Before giving the proof of Theorem II we need the following result which was first explicitly pointed out in [BT] with a very esoteric proof. Other proofs have been given in [K and N2]. We shall show that the result can be readily reduced to a much older result of Smulian, Eberlein and Grothendieck (see [D]); namely, the following theorem is true if $X$ is the space $C(K)$ of continuous functions on the compact Hausdorff space $K$ and if we identify $E$ with $K$ in the canonical way. 
THEOREM 5. Suppose that $F$ is a bounded subset of the Banach space $X$ that is relatively countably compact in the $\sigma(X, E)$ topology, where $E$ is the set of extreme points of the unit ball of $X^{*}$. Then $F$ is relatively compact in the weak topology.

ProOF. Let $\left\{z_{n}\right\}$ be a sequence in $F$ and suppose that $F$ is a subset of the unit ball of $X$ and $z_{0}$ is in $X$ and is a $\sigma(X, E)$ cluster point of $\left\{z_{n}\right\}$ but is not a weak cluster point. Let $Z=\left[\left\{z_{i} \mid i \geq 0\right\}\right]$. We may find $x_{j}^{*} \in X^{*},\left\|x_{j}\right\| \leq 1,1 \leq j \leq l$, $1 \leq m$ and $\varepsilon>0$ so that

$$
\bigcap_{1 \leq j \leq l}\left\{x|| x_{j}^{*}\left(x-z_{0}\right) \mid<\varepsilon\right\} \cap\left\{z_{i} \mid i \geq m\right\}=\varnothing .
$$

Denote by $z_{j}^{*}$ the restriction of $x_{j}^{*}$ to $Z$. The separable representation theorem (see [Cho]) says that we may find weak* Borel probability measures $\mu_{j}$ on the unit ball of $Z^{*}$ so that $\mu_{j}\left(E_{Z}\right)=1$ where $E_{Z}$ denotes the extreme points of the unit ball of $Z^{*}$ and $\mu_{j}$ represents $z_{j}$ for all $z$ in $Z, z_{j}^{*}(z)=\int z^{*}(z) d \mu_{j}$. We may find weak ${ }^{*}$ compact subsets $T_{j}$ of $E_{Z}$ such that $\mu_{j}\left(T_{j}\right)>1-\varepsilon / 4$. Let $T=\bigcup_{1 \leq j \leq l} T_{j}$. Define

$$
V=\bigcap_{1 \leq j \leq l}\left\{x|| \int_{T}\left(x-z_{0}\right) d \mu_{j} \mid<\frac{\varepsilon}{2} \text { for } 1 \leq j \leq l\right\} .
$$

If $z_{i}$ is in $V$ for some $i \geq m$ then for each $j$ we have that

$$
\frac{\varepsilon}{2}>\left|\int_{T}\left(z_{0}-z_{i}\right) d \mu_{j}\right| \geq\left|z_{j}^{*}\left(z_{0}-z_{i}\right)\right|-\int_{D}\left|z_{0}-z_{i}\right| d \mu_{j} \geq\left|z_{j}^{*}\left(z_{0}-z_{i}\right)\right|-\frac{\varepsilon}{2}
$$

where $D=E_{Z} \backslash T$. This is a contradiction. Thus $V \cap\left\{z_{i} \mid i \geq m\right\}=\varnothing$. Let $K=\left\{x^{*} \in X^{*} \mid\left\|x^{*}\right\| \leq 1\right.$ and $\left.x^{*} \mid Z \in T\right\}$. We shall show that $\left\{z_{i}|K| i \in \mathcal{N}\right\}$ is relatively weakly compact in $C(K)$. Choose any subsequence $\left\{y_{k}\right\}$ of $\left\{z_{i}\right\}$. There exists $x$ in $X$ that is a $\sigma(X, E)$ cluster point of $\left\{y_{k}\right\}$. Define for each $z^{*} \in T$

$$
K\left(z^{*}\right)=\left\{x^{*} \in K\left|x^{*}\right| Z=z^{*}\right\} .
$$

The extreme points of $K\left(z^{*}\right)$ are extreme points of the unit ball of $X^{*}$. Since each $y_{k}$ is constant on each $K\left(z^{*}\right)$ we have that $x$ is also constant on each $K\left(z^{*}\right)$ and we have that $x$ is a $\sigma(X, K)$ cluster point of $\left\{y_{k}\right\}$. From the first part of the proof we know that $\left\{z_{i}|K| i \in \mathcal{N}\right\}$ is relatively weakly compact in $C(K)$ and $z_{0}$ is a $\sigma(C(K), K)$ cluster point of $\left\{z_{i} \mid i \in \mathcal{N}\right\}$. Extend each measure $\mu_{j}$ to a measure $\nu_{j}$ on the unit ball of $X^{*}$ such that $\mu_{j}(T)=\nu_{j}(K)$. From the theorem above some subsequence of $\left\{z_{i} \mid i \in \mathcal{N}\right\}$ converges weakly to $z_{0}$ in $C(K)$. We also know that for $i \geq m$

$$
\sup _{1 \leq j \leq l}\left|\int_{K}\left(z_{i}-z_{0}\right) d \nu_{j}\right|=\sup _{1 \leq j \leq l}\left|\int_{T}\left(z_{i}-z_{0}\right) d \mu_{j}\right|>\frac{\varepsilon}{2}
$$

which is a contradiction. This proves that any countable subset of $F$ has a weak cluster point and the theorem above shows that $F$ is relatively weakly compact.

Analogous to [Cho] (see also [Chr]), we define a strategy to be a function $s$ from the nonempty open subsets $\mathcal{U}$ of $T$ to $\mathcal{U}$ with the following property:

(*) $s(U) \subseteq U$ and if $\left\{U_{n}\right\}$ is a sequence of open sets such that $U_{n+1} \subseteq s\left(U_{n}\right)$ and $t_{n} \in U_{n}$, then the sequence $\left\{t_{n}\right\}$ has a cluster point in $\bigcap_{n=1}^{\infty} U_{n}$.

It is easy to see that Cech-complete spaces have property $(*)$. 
We reformulate Theorem II in the following way:

THEOREM 6. Let $T$ be a topological space satisfying (*) and $f: T \rightarrow X$ be a $\sigma(X, E)$ continuous function. Then, there exists a dense $G_{\delta}$ subset $G$ of $T$ such that $f$ is continuous with respect to the norm topology at each point of $G$.

ProOF. (Exactly as in [S1].) As usual, define $T_{n}=\bigcup\{U \mid U$ open, $\operatorname{diam} f(U)$ $\left.\leq 2^{-n}\right\}$. Each $T_{n}$ is open and if each $T_{n}$ is dense then $G=\bigcap_{n=1}^{\infty} T_{n}$ is the desired set. Also, for each $m$,

$$
\{t \mid\|f(t)\| \leq m\}=\bigcap\left\{t \mid e^{*}(f(t)) \leq m, e^{*} \in E\right\}
$$

is a closed set. Since $T$ is a Baire space we may assume there exist a nonempty open set $U_{0,0}$ and constants $C>0, \delta>0$ such that

$$
\|f(t)\| \leq C \text { for all } t \in U_{0,0}
$$

and $\operatorname{diam} f(U)>\delta$ for all $\varnothing \neq U$ open, $U \subseteq U_{0,0}$.

The proof proceeds as in the usual "Cantor-space" construction. Since

$$
\operatorname{diam} f\left(U_{0,0}\right)>\delta
$$

and the set of extreme points are norming, we may choose an extreme point $e_{0,0}^{*}$ and $r_{0,0}$ real such that

$$
\begin{gathered}
s\left(U_{0,0}\right) \cap\left\{t \mid e_{0,0}^{*}(f(t))<r_{0,0}\right\}=U_{1,0}, \\
s\left(U_{0,0}\right) \cap\left\{t \mid e_{0,0}^{*}(f(t))>r_{0,0}+\delta\right\}=U_{1,1}
\end{gathered}
$$

are both nonempty. Repeat this argument for $s\left(U_{1,0}\right)$ and $s\left(U_{1,1}\right)$. We obtain a sequence of extreme points

$$
\left\{e_{n, i}^{*} \mid 0 \leq n<\infty, 0 \leq i<2^{n}-1\right\}
$$

a sequence of real numbers

$$
\left\{r_{n, i} \mid 0 \leq n<\infty, 0 \leq i<2^{n}-1\right\},
$$

and a sequence of nonempty open subsets

$$
\left\{U_{n, i} \mid 0 \leq n<\infty, 0 \leq i<2^{n}-1\right\}
$$

of $T$ such that

$$
U_{n+1,2 i} \cap U_{n+1,2 i+1} \subseteq s\left(U_{n, 1}\right)
$$

and

$$
\sup _{t \in U_{n+1,2 i}} e_{n, i}^{*}(f(t)) \leq r_{n, i}, \quad \inf _{t \in U_{n+1,2 i+1}} e_{n, i}^{*}(f(t)) \geq r_{n, i}+\delta
$$

Define

$$
S=\bigcap_{n=0}^{\infty} \bigcup_{i=0}^{2^{n}-1} s\left(U_{n, i}\right) .
$$

It is easy to see (exactly as in the classical Bolzano-Weierstrass theorem) that each sequence in $S$ has a cluster point in $S ; f(S)$ has the same property with respect to the $\sigma(X, E)$ topology. By the result above, $f(S)$ is weakly compact. Let $Y$ be the smallest, norm-closed linear subspace of $X^{*}$ containing the vectors $\left\{e_{n, i}^{*}\right\}$; $Y$ is a separable Banach space. The induced function from $S$ to $Y^{*}$ is $\sigma\left(Y^{*}, Y\right)$ 
continuous. Since $f(S)$ is bounded and weakly compact it is also $\sigma\left(Y^{*}, Y\right)$ compact. The $\sigma\left(Y^{*}, Y\right)$ topology on $f(S)$ is metrizable, thus $f(S)$ is weakly separable and by the Hahn-Banach theorem it is also norm-separable. This is a contradiction because $S$ contains an uncountable set $S_{0}$ (indexed by the "branches" of $\{(n, i)$ | $\left.\left.n=0, \ldots, 0 \leq i<2^{n}\right\}\right)$ such that for two distinct points $s$ and $t$ in $S_{0}$,

$$
\|f(s)-f(t)\|_{Y^{*}} \geq \sup _{n, i}\left\{\left|e_{n, i}^{*}(f(s)-f(t))\right|\right\} \geq \delta .
$$

We mention that the above proof also works for multivalued maps: suppose that $T$ and $X$ are as above and that $F: T \rightarrow X$ is an upper semicontinuous and compact valued map (understood that $X$ has the $\sigma(X, E)$ topology). Then there exists a function $f: T \rightarrow X$ and a dense $G_{\delta}$ subset $G$ of $T$ such that $f(t) \in F(t)$ for all $t$ and $f$ is continuous at each point of $G$. We may assume that $F$ is minimal (Zorn's Lemma); the proof then proceeds exactly as above (by $F(A)$ we mean $\bigcup\{F(t) \mid t \in A\})$. Note that if $F$ is minimal, $U, V$ are open subsets of $T$ and $X$, respectively, and $F(U) \cap V \neq \varnothing$, then there exists an open $W$ with $U \supseteq W \neq \varnothing$, such that $F(W) \subseteq V$; one need only bear this in mind in the choice of $U_{n, i}$.

ACKNOWLEDGMENT. The author would like to thank M. Bauer for preparing the computer program in which this paper was written.

\section{BIBLIOGRAPHY}

[B] N. Bourbaki, Topologie générale, Hermann, Paris, 1951.

[BT] J. Bourgain and M. Talagrand, Compacité extrémale, Proc. Amer. Math. Soc. 80 (1980), 68-70.

[Cho] G. Choquet, Lectures on analysis, Benjamin, New York, 1969.

[Chr] J. P. R. Christensen, Joint continuity of separately continuous functions, Proc. Amer. Math. Soc. 82 (1981), 455-461.

[D] M. M. Day, Normed linear spaces, Springer, Berlin, 1973.

[Deb] G. Debs, Espaces de Baire et espaces $k$-analytiques de fonctions continue, Mathematika 32 (1985), 218-228.

[Dev] R. Deville, Parties faiblement de Baire dans les espaces de Banach (to appear).

[E] R. Engelking, General topology, PWN, Warszawa, 1977.

[K] S. S. Kurana, Pointwise compactness on extreme points, Proc. Amer. Math. Soc. 83 (1981), 347-348.

[N1] I. Namioka, Separate continuity and joint continuity, Pacific J. Math. 51 (1974), 515-531.

[N2] _ A simplification of Kurana's proof, Rainwater Seminar, University of Washington.

[SR] J. Saint Raymond, Jeux topologiques et espaces de Namioka, Proc. Amer. Math. Soc. 87 (1983), 499-504.

[S1] C. Stegall, The duality between Asplund spaces and spaces with the Radon-Nikodym property, Israel J. Math. 29 (1978), 408-412.

[S2] _ _ A result concerning the weak topology generated by the extreme linear functionals, Séminaire d'Analyse Fonctionelle, Université de Paris VII, 1983-84.

[S3] _ Applications of descriptive topology in functional analysis, Universität Linz, 1985.

[T1] M. Talagrand, Espaces de Banach faiblement $k$-analytique, Ann. of Math. 110 (1979), 407-438.

[T2] _ Jeux topologiques et espaces de Namioka (to appear).

Institut Für MAthematik, Johannes Kepler UniVersität LinZ, A-4040 LinZ, AUSTRIA 